\title{
Fish larvae from the Gulf of California*
}

\author{
GERARDO ACEVES-MEDINA ${ }^{1}$, S. PATRICIA A. JIMÉNEZ-ROSENBERG ${ }^{1}$, \\ ALEJANDRO HINOJOSA-MEDINA ${ }^{1}$, RENÉ FUNES-RODRIGUEZ ${ }^{1}$, RICARDO J. \\ SALDIERNA $^{1}$, DANIEL LLUCH-BELDA ${ }^{1}$, PAUL E. SMITH ${ }^{2}$ and WILLIAM WATSON ${ }^{2}$ \\ ${ }^{1}$ INP-Centro Interdisciplinario de Ciencias Marinas, Depto. de Plancton y Ecología Marina, Apdo. Postal 592, La Paz, \\ Baja California Sur, México, C.P. 23000. E-mail: aceves651@ prodigy.net.mx \\ ${ }^{2}$ NOAA/NMFS, Southwest Fisheries Science Center, La Jolla, California 92038, USA.
}

\begin{abstract}
SUMMARY: Taxonomic composition of fish larvae was analysed from 464 plankton samples obtained during 10 oceanographic surveys in the Gulf of California between 1984 and 1988. We identified 283 taxa: 173 species, 57 genera, and 53 families. Tropical and subtropical species predominated except during the winter, when temperate-subarctic species were dominant. The most abundant species were the mesopelagic Benthosema panamense, Triphoturus mexicanus and Vinciguerria lucetia, but the coastal pelagic species Engraulis mordax, Opisthonema spp., Sardinops caeruleus and Scomber japonicus were also prominent. The taxonomic composition of the ichthyoplankton shows the seasonality of the Gulf as well as environmental changes that occurred between the 1984-1987 warm period and the 1956-1957 cool period previously reported. The presence of E. mordax larvae as one of the most abundant species in the Gulf provides evidence of the reproduction of this species two years before the development of the northern anchovy fishery and the decline of the sardine fishery in the Gulf of California.
\end{abstract}

Key words: fish larvae, ichthyoplankton, Gulf of California.

RESUMEN: LaRvas de PeCes del Golfo de CALIFornia. - Se analizó la composición taxonómica de las larvas de peces capturadas en 464 arrastres de plancton obtenidos durante 10 cruceros oceanográficos en el Golfo de California entre 1984 y 1988. Se identificaron 283 taxones: 173 especies, 57 géneros y 53 familias. Los organismos de afinidad tropical y subtropical fueron los más numerosos excepto durante el invierno, periodo en el que las especies templado-subárticas fueron dominantes. Las especies más abundantes fueron los mesopelágicos Benthosema panamense, Triphoturus mexicanus y Vinciguerria lucetia, pero las especies pelágico-costeras como Engraulis mordax, Opisthonema spp., Sardinops caeruleus y Scomber japonicus fueron también importantes por el número de organismos recolectados. La composición taxonómica del ictioplancton refleja la variabilidad estacional del Golfo, así como los cambios ocurridos entre el periodo cálido de 19841987 y el periodo frío de 1956-1957 previamente registrado en la literatura. La presencia de larvas de E. mordax como una de las especies más abundantes en el Golfo, aporta evidencia de la reproducción de esta especie dos años antes del desarrollo de la pesquería de la anchoveta norteña y el desplome de la pesquería de sardina del Golfo de California.

Palabras clave: larvas de peces, ictioplancton, Golfo de California.

\section{INTRODUCTION}

The Gulf of California is a semi-enclosed system bordered on the west by the Baja California peninsula and on the east by the Mexican mainland. To

*Received July 4, 2001. Accepted July 1, 2002. the south it connects to the Pacific Ocean through a $200 \mathrm{~km}$ wide mouth (Fig. 1). The Gulf has one of the most diverse and highly endemic ichthyofaunas of the eastern Pacific, which supports its designation as a separate zoogeographic province from the Panamic province, in which it is usually included due to its high proportion of tropical species (Walker, 1960; 
Thomson et al., 1979). Although Ekman (1953) first suggested that the Gulf of California ecosystem represents a distinct zoogeographic province, it was not until Briggs (1974) that it received formal status as the Cortez province, which borders to the south with the Mexican province on a line drawn between Cabo San Lucas and Mazatlán. The Cortez province is characterised by a relict Pleistocene temperate-subarctic fauna located north of Tiburón and Angel de la Guarda Islands that was apparently native from the San Diego province and by a tropical-subtropical fauna found primarily in the south. Both the northern and southern faunas merge in the central region, forming a zone of high spatial and temporal faunistic contrast (Castro-Aguirre et al., 1995).

Even though the fish fauna of the Gulf of California is one of the best studied of the eastern Pacific, only a small number of studies have addressed the early life histories of the fishes. Most previous ichthyoplankton studies have been oriented towards species with commercial value, such as the Pacific sardine and the northern anchovy (Green-Ruiz and Hinojosa-Corona, 1997). Except for the work of Moser et al. (1974), no one has addressed the composition of the ichthyoplankton of the Gulf, primarily because little was known of the taxonomy of the

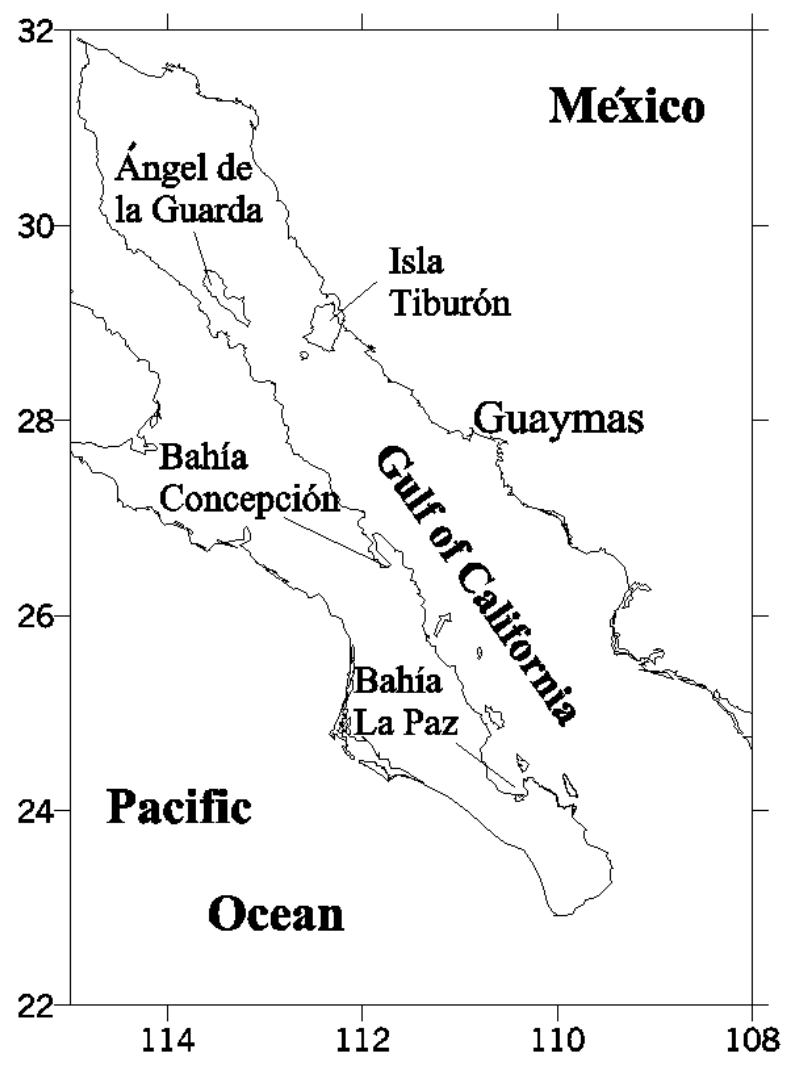

FIG. 1. - Study area. fish larvae. Richards (1985) and Kendall and Matarese (1994) reported that larvae have been described for nearly $10 \%$ of the fish species worldwide, but in the eastern tropical Pacific region this proportion is even lower, as reflected in the work of Moser et al. (1974), who found that more than $60 \%$ of the fish larvae of the Gulf were identifiable only to family. Since 1951 the California Cooperative Oceanic Fisheries Investigations program (Cal$\mathrm{COFI}$ ) has conducted ichthyoplankton research in the California current region, allowing more detailed analyses of ichthyoplankton composition and abundance in the California and western coast of Baja California (e.g. Loeb et al., 1983; Moser et al., 1987; Moser and Smith, 1993), and a wide taxonomic knowledge that includes many species found in the Gulf of California (Moser, 1996).

Progress in the identification of the fish larvae inhabiting the Gulf of California has allowed a more detailed analysis of the ichthyoplankton in terms of species composition, distribution patterns, species assemblages, and how fish larvae are affected by environmental changes, resulting in a better understanding of the ecosystem. This work is the first in a series whose objective is to characterise the larval fish communities of the Gulf of California. Here we draw up the taxonomic list and compare our findings with those of Moser et al. (1974).

\section{MATERIALS AND METHODS}

Ten oceanographic surveys were conducted in the Gulf of California during April, July and November-December 1984, April 1985, June, July and August 1986, September and November 1987, and February 1988 (Fig. 2). Each cruise was named with the acronym GOLCA (for Golfo de California) and four digits, the first two indicating the year and the last two the month of sampling. Therefore GOLCA 8404 refers to sampling in the Gulf of California in April 1984.

A total of 464 plankton samples were obtained with oblique tows using Bongo nets with 333- and $505-\mu \mathrm{m}$ mesh size following the sampling methodology detailed by Smith and Richardson (1979). Fish larvae were removed from samples and stored in $2 \%$ formalin buffered with a saturated solution of sodium borate. Only the larvae from the $505-\mu \mathrm{m}$ mesh net were analysed; these were identified to the lowest possible taxon using Moser (1996). Larvae of each taxon were counted and their abundances stan- 




FIG. 2. - Sampling stations.

dardised to $10 \mathrm{~m}^{2}$ of sea surface following Smith and Richardson (1979). The taxonomic organization of fish larvae is that of Eschmeyer et al. (1983) and Moser (1996); species are listed alphabetically within each family. Habitat and affinity data for each species were taken from Moser (1996). For purposes of examining the seasonal distribution of each taxon, cruises occurring in April (GOLCA 8404 and 8504) were assigned to the spring period, June through September to the summer period (GOLCA 8407, 8606, 8608 and 8709), November and Decem- ber to the fall period (GOLCA 8412, 8611 and 8711), and February to the winter period (GOLCA 8802).

\section{RESULTS}

A total of 283 taxa were identified, of which 173 were identified to species, 57 to genus, and 53 to family (Table 1). In most cases, larvae that could not be identified to species were identified to types 
TABLE 1. - Systematic list of the fish larvae collected in the Gulf of California during 1984-1988 with the percent abundance (\%). Faunistic affinity (FA): tropical (tr); subtropical (st); temperate (tm); subarctic (sa); wide distribution in the eastern Pacific (wd). Habitat (Hab): shallow demersal (sd); deep demersal (dd); coastal pelagic (cp); ocean epipelagic (op); mesopelagic (mp); and bathypelagic (bp).

\begin{tabular}{|c|c|c|c|c|c|}
\hline Taxon & $\%$ & (FA)-(Hab) & Taxon & $\%$ & (FA)-(Hab) \\
\hline O. Elopiformes & & & F. Melanostomiidae & & \\
\hline F. Elopidae & & & Melanostomiidae type 1 & $<0.1$ & $-(\mathrm{mp})$ \\
\hline Elops affinis Regan 1909 & $<0.1$ & $(\mathrm{tr} / \mathrm{st})-(\mathrm{cp})$ & O. Aulopiformes & & \\
\hline O. Albuliformes & & & S.O. Aulopoidei & & \\
\hline F. Albulidae & & & F. Aulopidae & & \\
\hline Albula type 1 (A vulpes?) & 0.7 & $(\mathrm{tr})-(\mathrm{sd})$ & Aulopus bajacali Parin \& Kotlyar 1984 & $<0.1$ & $(\mathrm{tr})-(\mathrm{dd})$ \\
\hline O. Notacanthiformes & & & F. Scopelarchidae & & \\
\hline F. Notacanthidae & & & Scopelarchoides nicholsi (Parr 1929) & $<0.1$ & $(\mathrm{tr})-(\mathrm{mp})$ \\
\hline Notacanthidae & $<0.1$ & $-(\mathrm{dd})$ & Scopelarchus guentheri Alcock 1896 & $<0.1$ & $(\mathrm{tr} / \mathrm{st})-(\mathrm{mp})$ \\
\hline O. Anguilliformes & & & Scopelarchidae type 1 & $<0.1$ & $-(\mathrm{mp})$ \\
\hline F. Muraenidae & & & S.O. Alepisauroidei & & \\
\hline Anarchias type 1 & $<0.1$ & $(\mathrm{sd})$ & F. Synodontidae & & \\
\hline Gymnothorax type 1 & $<0.1$ & $(\mathrm{tr} / \mathrm{st})-(\mathrm{sd})$ & Synodus lucioceps (Ayres 1855) & $<0.1$ & $(\mathrm{tm})-(\mathrm{sd})$ \\
\hline Uropterygius type 1 & $<0.1$ & (sd) & Synodus spp. & 0.1 & (tr)-(sd) \\
\hline Muraenidae type 1 & $<0.1$ & (sd) & F. Paralepididae & & \\
\hline F. Ophichthidae & & & Lestidiops neles (Harry 1953)s & $<0.1$ & $(\mathrm{tr})-(\mathrm{mp})$ \\
\hline Myrophis vafer Jordan \& Gilbert 1882 & $<0.1$ & $(\mathrm{tm} / \mathrm{st})-(\mathrm{sd})$ & Lestidiops type 1 & $<0.1$ & $-(\mathrm{mp})$ \\
\hline Ophichthus triserialis (Kaup 1856) & $<0.1$ & $(\mathrm{tr} / \mathrm{st})-(\mathrm{sd})$ & O. Myctophiformes & & \\
\hline Ophichthus zophochir & & & F. Neoscopelidae & & \\
\hline (Jordan \& Gilbert 1882) & 0.1 & $(\mathrm{tr} / \mathrm{st})-(\mathrm{sd})$ & Neoscopelidae type 1 & $<0.1$ & $-(b p)$ \\
\hline Ophichthus type 1 & $<0.1$ & $-(\mathrm{sd})$ & F. Myctophidae & & \\
\hline Ophichthidae type 1 & $<0.1$ & $-(\mathrm{sd})$ & Ceratoscopelus townsendi & $<0.1$ & $(w d)-(m p)$ \\
\hline F. Congridae & & & (Eigenmann \& Eigenmann 1889) & & \\
\hline Ariosoma gilberti (Ogilby 1898) & $<0.1$ & $(\mathrm{tr} / \mathrm{st})-(\mathrm{sd})$ & Diaphus pacificus Parr 1931 & 0.2 & $(\mathrm{tr})-(\mathrm{mp})$ \\
\hline Bathycongrus macrurus (Gilbert 1891) & $<0.1$ & $(\mathrm{tr})-(\mathrm{sd})$ & Lampanyctus parvicauda Parr 1931 & $<0.1$ & $(\mathrm{tr})-(\mathrm{mp})$ \\
\hline Chiloconger type 1 & $<0.1$ & $(\mathrm{tr} / \mathrm{st})-(\mathrm{sd})$ & Nannobrachium idostigma (Parr 1931) & $<0.1$ & $(\mathrm{tr} / \mathrm{st})-(\mathrm{mp})$ \\
\hline Gnathophis cinctus (Garman 1899) & $<0.1$ & $(\mathrm{tm} / \mathrm{st})-(\mathrm{sd})$ & Nannobrachium type 1 & $<0.1$ & $-(\mathrm{mp})$ \\
\hline Paraconger type 1 & $<0.1$ & $(\mathrm{tr} / \mathrm{st})-(\mathrm{sd})$ & Triphoturus mexicanus (Gilbert 1890) & 7.1 & (st)-(mp) \\
\hline Congridae type 1 & $<0.1$ & $-(\mathrm{sd})$ & Benthosema panamense (Tåning 1932) & 34.2 & $(\mathrm{tr})-(\mathrm{sd})$ \\
\hline F. Derichthyidae & & & Diogenichthys laternatus (Garman 1899) & 3.5 & $(\mathrm{tr})-(\mathrm{mp})$ \\
\hline Derichthyidae type 1 & $<0.1$ & $-(\mathrm{mp})$ & Gonichthys tenuiculus (Garman 1899) & $<0.1$ & $(\mathrm{tr} / \mathrm{st})-(\mathrm{mp})$ \\
\hline F. Nemichthyidae & & & Hygophum atratum (Garman 1899) & 0.2 & $(\mathrm{tr})-(\mathrm{mp})$ \\
\hline Nemichthyidae type 1 & $<0.1$ & $-(\mathrm{mp})$ & Myctophidae type 1 & $<0.1$ & $-(\mathrm{mp})$ \\
\hline F. Serrivomeridae & & & O. Gadiformes & & \\
\hline Serrivomeridae & $<0.1$ & (bp) & F. Bregmacerotidae & & \\
\hline F. Nettastomatidae & & & Bregmaceros bathymaster & 0.2 & $(\mathrm{tr} / \mathrm{st})-(\mathrm{cp})$ \\
\hline Hoplunnis sicarius (Garman 1899) & $<0.1$ & $(\mathrm{tr})-(\mathrm{sd})$ & Jordan \& Bollman 1890 & & \\
\hline Hoplunnis type 1 & $<0.1$ & $(\mathrm{tr})-(\mathrm{sd})$ & Bregmaceros type 1 & $<0.1$ & $(\mathrm{tr})-(\mathrm{cp})$ \\
\hline F. Cyematidae & & & Bregmacerotidae type 1 & $<0.1$ & $-(\mathrm{cp})$ \\
\hline Cyematidae type 1 & $<0.1$ & $-(\mathrm{mp})$ & F. Macrouridae & & \\
\hline O. Clupeiformes & & & Caelorinchus scaphopsis (Gilbert 1890) & $<0.1$ & $(\mathrm{tm})-(\mathrm{bp})$ \\
\hline F. Clupeidae & & & Nezumia type 1 & $<0.1$ & (bp) \\
\hline Etrumeus teres (DeKay 1842) & 1.0 & $(w d)-(c p)$ & F. Moridae & & \\
\hline $\begin{array}{l}\text { Harengula thrissina } \\
\quad \text { (Jordan \& Gilbert 1882) }\end{array}$ & 0.1 & $(\mathrm{tr})-(\mathrm{cp})$ & $\begin{array}{l}\text { Physiculus nematopus Gilbert } 1890 \\
\text { F. Merlucciidae }\end{array}$ & $<0.1$ & $(\mathrm{tr})-(\mathrm{mp})$ \\
\hline Opisthonema sp. (libertate?) & 5.5 & $(\mathrm{tr})-(\mathrm{cp})$ & Merluccius productus (Ayres 1855) & 0.7 & $(\mathrm{tm} / \mathrm{sa})-(\mathrm{dd})$ \\
\hline Sardinops caeruleus (Girard 1856) & 3.6 & $(\mathrm{tm} / \mathrm{sa})-(\mathrm{cp})$ & O. Ophidiiformes & & \\
\hline Clupeidae & $<0.1$ & $-(\mathrm{cp})$ & S.O. Ophidioidei & & \\
\hline F. Engraulidae & & & F. Ophidiidae & & \\
\hline Engraulis mordax Girard 1854 & 17.6 & $(\mathrm{tm})-(\mathrm{cp})$ & Cherublemma emmelas (Gilbert 1890) & $<0.1$ & $(\mathrm{tr})-(\mathrm{dd})$ \\
\hline Engraulidae type 1 & 2.5 & $-(\mathrm{cp})$ & Chilara taylori (Girard 1958) & $<0.1$ & $(\mathrm{tm} / \mathrm{sa})-(\mathrm{dd})$ \\
\hline O. Salmoniformes & & & Lepophidium negropinna & $<0.1$ & $(\mathrm{tr})-(\mathrm{sd})$ \\
\hline S.O. Argentinoidei & & & Hildebrand \& Barton 1949 & & \\
\hline F. Argentinidae & & & Lepophidium stigmatistium Gilbert 1890 & $<0.1$ & $(\mathrm{st})-(\mathrm{dd})$ \\
\hline Argentina sialis Gilbert 1890 & 0.1 & $(\mathrm{tm})-(\mathrm{dd})$ & Lepophidium type 1 & $<0.1$ & $(\mathrm{tr})-(\mathrm{sd})$ \\
\hline F. Bathylagidae & & & Ophidion scrippsae (Hubbs 1916) & $<0.1$ & $(\mathrm{tm})-(\mathrm{sd})$ \\
\hline Bathylagus pacificus Gilbert 1890 & $<0.1$ & $(\mathrm{tm} / \mathrm{sa})-(\mathrm{mp})$ & Ophidion type 1 & $<0.1$ & $-(\mathrm{sd})$ \\
\hline Bathylagus wesethi Bolin 1938 & $<0.1$ & $(\mathrm{tm})-(\mathrm{mp})$ & Ophidiidae & $<0.1$ & \\
\hline Leuroglossus stilbius Gilbert 1890 & 1.6 & $(\mathrm{tm})-(\mathrm{mp})$ & F. Carapidae & & \\
\hline F. Microstomatidae & & & Encheliophis dubis (Putman 1874) & $<0.1$ & $(\mathrm{tr})-(\mathrm{sd})$ \\
\hline Microstomatidae type 1 & $<0.1$ & $-(\mathrm{mp})$ & Encheliophis type 1 & $<0.1$ & \\
\hline O. Stomiiformes & & & Echiodon exsilium Rosenblatt 1961 & $<0.1$ & $(\mathrm{tr})-(\mathrm{dd})$ \\
\hline F. Gonostomatidae & & & Carapidae & $<0.1$ & \\
\hline Diplophos proximus Parr 1931 & $<0.1$ & $(\mathrm{tr})-(\mathrm{mp})$ & F. Bythitidae & & \\
\hline F. Phosichthyidae & & & Bythitidae type 1 & $<0.1$ & \\
\hline Ichthyococcus irregularis & & & O. Lophiiformes & & \\
\hline Rechnitzer \& Böhlke 1958 & $<0.1$ & $(w d)-(m p)$ & S.O. Lophioidei & & \\
\hline Vinciguerria lucetia (Garman 1899) & 6.8 & $(\mathrm{tr})-(\mathrm{mp})$ & F. Lophiidae & & \\
\hline F. Stomiidae & & & Lophiodes spilurus (Garman 1899) & $<0.1$ & $(w d)-(d d)$ \\
\hline Stomias atriventer Garman 1899 & $<0.1$ & $(\mathrm{tr} / \mathrm{st})-(\mathrm{mp})$ & Lophiodes type 1 & $<0.1$ & \\
\hline
\end{tabular}


TABLE 1 (Cont.). - Systematic list of the fish larvae collected in the Gulf of California during 1984-1988 with the percent abundance (\%). Faunistic affinity (FA): tropical (tr); subtropical (st); temperate (tm); subarctic (sa); wide distribution in the eastern Pacific (wd). Habitat (Hab): shallow demersal (sd); deep demersal (dd); coastal pelagic (cp); ocean epipelagic (op); mesopelagic (mp); and bathypelagic (bp).

\begin{tabular}{|c|c|c|c|c|c|}
\hline Taxon & $\%$ & (FA)-(Hab) & Taxon & $\%$ & $(\mathrm{FA})-(\mathrm{Hab})$ \\
\hline Lophiidae type 1 & $<0.1$ & & Scorpaenidae & $<0.1$ & \\
\hline S.O. Antennarioidei & & & F. Triglidae & & \\
\hline F. Antennariidae & & & Bellator loxias (Jordan 1897) & $<0.1$ & $(\mathrm{tr})-(\mathrm{sd})$ \\
\hline Antennarius avalonis Jordan \& Starks 1907 & $<0.1$ & $(\mathrm{tr} / \mathrm{st})-(\mathrm{sd})$ & Prionotus ruscarius Gilbert \& Starks 1904 & $<0.1$ & $(\operatorname{tr})-(\mathrm{sd})$ \\
\hline Antennarius type 1 & $<0.1$ & & Prionotus stephanophrys & $<0.1$ & (wd)-(sd) \\
\hline S.O. Ogcocephalioidei & & & Lockington 1881 & & \\
\hline F. Ogcocephalidae & & & Triglidae & $<0.1$ & $-(\mathrm{sd})$ \\
\hline Zalieutes elater (Jordan \& Gilbert 1882) & $<0.1$ & $(\mathrm{tr} / \mathrm{st})-(\mathrm{dd})$ & O. Perciformes & & \\
\hline S.O. Ceratioidei & & & S.O. Percoidei & & \\
\hline F. Melanocetidae & & & F. Serranidae & & \\
\hline Melanocetidae type 1 & $<0.1$ & $(\mathrm{tr} / \mathrm{st})-(\mathrm{bp})$ & Hemanthias signifer (Garman 1899) & $<0.1$ & (tr)-(sd) \\
\hline F. Oneirodidae & & & Hemanthias spp. & $<0.1$ & $(\operatorname{tr})-(\mathrm{sd})$ \\
\hline Oneirodes type 1 & $<0.1$ & $-(b p)$ & Mycteroperca spp. & $<0.1$ & $(\mathrm{tr} / \mathrm{st})-(\mathrm{sd})$ \\
\hline Oneirodidae type 1 & $<0.1$ & $-(\mathrm{bp})$ & Epinephelus spp. & $<0.1$ & $(\mathrm{tr} / \mathrm{st})-(\mathrm{sd})$ \\
\hline F. Gigantactinidae & & & Diplectrum spp. & 0.1 & $(\operatorname{tr} / \mathrm{st})-(\mathrm{sd})$ \\
\hline Gigantactis type 1 & $<0.1$ & $-(b p)$ & Paralabrax maculatofasciatus & $<0.1$ & $(\mathrm{tm} / \mathrm{st})-(\mathrm{sd})$ \\
\hline F. Linophrynidae & & & (Steindechner 1868) & & \\
\hline Borophryne apogon Regan 1925 & $<0.1$ & $-(\mathrm{tr})-(\mathrm{bp})$ & Serranus spp. & 0.2 & $(\mathrm{tr} / \mathrm{st})-(\mathrm{sd})$ \\
\hline O. Atheriniformes & & & Pronotogrammus multifasciatus Gill 1863 & $3<0.1$ & (tr)-(sd) \\
\hline F. Atherinidae & & & Paranthias colonus (Valenciennes 1846) & $<0.1$ & (tr)-(sd) \\
\hline Atherinidae type 1 & $<0.1$ & $-(\mathrm{cp})$ & Pseudogramma thaumasium (Gilbert 1890) & $<0.1$ & $(\mathrm{tr})-(\mathrm{sd})$ \\
\hline O. Beloniformes & & & Serranidae & $<0.1$ & $-(\mathrm{sd})$ \\
\hline F. Hemiramphidae & & & F. Priacanthidae & & \\
\hline Hyporhamphus rosae & $<0.1$ & $(\mathrm{tr} / \mathrm{st})-(\mathrm{cp})$ & Pristigenys serrula (Gilbert 1891) & $<0.1$ & $(w d)-(s d)$ \\
\hline (Jordan \& Gilbert 1880) & & & Priacanthidae type 1 & $<0.1$ & $-(\mathrm{sd})$ \\
\hline Oxyporhamphus micropterus & $<0.1$ & $(\operatorname{tr} / \mathrm{st})-(\mathrm{cp})$ & F. Apogonidae & & \\
\hline (Valenciennes 1847) & & & Apogon retrosella (Gill 1863) & $<0.1$ & $(\mathrm{tr})-(\mathrm{sd})$ \\
\hline Hemiramphidae type 1 & $<0.1$ & & Apogon spp. & $<0.1$ & $-(\mathrm{sd})$ \\
\hline F. Exocoetidae & & & Apogonidae type 1 & $<0.1$ & $-(\mathrm{sd})$ \\
\hline Cheilopogon pinnatibarbatus(Cooper 1863) & $<0.1$ & $(\mathrm{tm} / \mathrm{sa})-(\mathrm{cp})$ & F. Carangidae & & \\
\hline Fodiator acutus (Günther 1866) & $<0.1$ & $(\mathrm{tr} / \mathrm{st})-(\mathrm{cp})$ & Alectis ciliaris (Bloch 1787) & $<0.1$ & $(\operatorname{tr})-(\mathrm{cp})$ \\
\hline Hirundichthys rondeletii & $<0.1$ & $(\mathrm{tr} / \mathrm{st})-(\mathrm{cp})$ & Caranx caballus Günther 1868 & 0.3 & $(\mathrm{tr} / \mathrm{st})-(\mathrm{cp})$ \\
\hline (Valenciennes 1846) & & & Caranx sexfasciatusQuoy \& Gaimard 1825 & $5<0.1$ & $(\operatorname{tr})-(\mathrm{cp})$ \\
\hline Hirundichthys spp. & $<0.1$ & $(\mathrm{tr} / \mathrm{st})-(\mathrm{cp})$ & Caranx spp. & $<0.1$ & $-(\mathrm{cp})$ \\
\hline Prognichthys tringa Breder 1928 & $<0.1$ & $(\operatorname{tr})-(\mathrm{cp})$ & Chloroscombrus orqueta & $<0.1$ & $(\mathrm{tr} / \mathrm{st})-(\mathrm{cp})$ \\
\hline Exocoetidae type 1 & $<0.1$ & $(\operatorname{tr} / \mathrm{st})-(\mathrm{cp})$ & Jordan \& Gilbert 1883 & & \\
\hline O. Lampridiformes & & & Decapterus spp. & $<0.1$ & $(\operatorname{tr})-(\mathrm{cp})$ \\
\hline F. Trachipteridae & & & Elagatis bipinnulata & $<0.1$ & (tr)-(cp) \\
\hline Zu cristatus (Bonelli 1819) & $<0.1$ & $(\mathrm{tr} / \mathrm{st})-(\mathrm{cp})$ & (Quoy \& Gaimard 1825) & & \\
\hline Trachipteridae type 1 & $<0.1$ & & Gnathonodon speciosus (Forskål 1775) & $<0.1$ & (tr)-(cp) \\
\hline O. Beryciformes & & & Oligoplites saurus Gill 1863 & $<0.1$ & $(\operatorname{tr})-(\mathrm{cp})$ \\
\hline S.O. Berycoidei & & & Oligoplites type 1 & 0.2 & $(\mathrm{tr} / \mathrm{st})-(\mathrm{cp})$ \\
\hline F. Holocentridae & & & Selar crumenophthalmus (Bloch 1793) & 0.1 & $(\mathrm{tr} / \mathrm{st})-(\mathrm{cp})$ \\
\hline Myripristis leiognathos & $<0.1$ & $(\mathrm{tr})-(\mathrm{sd})$ & Selene brevoortii (Gill 1863) & $<0.1$ & $(\mathrm{tr} / \mathrm{st})-(\mathrm{cp})$ \\
\hline Valenciennes 1855 & & & Selene peruviana (Guichenot 1866) & $<0.1$ & $(\operatorname{tr})-(\mathrm{cp})$ \\
\hline Myripristis spp. & $<0.1$ & $(\mathrm{tr} / \mathrm{st})-(\mathrm{cp})$ & Selene spp. & $<0.1$ & $(\operatorname{tr} / \mathrm{st})-(\mathrm{cp})$ \\
\hline S.O. Stephanoberycoidei & & & Seriola lalandi Valenciennes 1833 & $<0.1$ & (wd)-(cp) \\
\hline F. Melamphaidae & & & Seriola spp. & $<0.1$ & $-(\mathrm{cp})$ \\
\hline Melamphaes spp. & $<0.1$ & $-(\mathrm{mp})$ & Trachinotus rhodopus Gill 1863 & $<0.1$ & (tr)-(cp) \\
\hline Scopelogadus bispinosus (Gilbert 1915) & $<0.1$ & (tr)-(mp) & Trachurus symmetricus (Ayres 1855) & $<0.1$ & $(\mathrm{tm} / \mathrm{sa})-(\mathrm{op})$ \\
\hline F. Mirapinnidae & & & Carangidae type 1 & $<0.1$ & $-(\mathrm{cp})$ \\
\hline Mirapinnidae type 1 & $<0.1$ & $-(\mathrm{mp})$ & F. Nematistiidae & & \\
\hline O. Syngnathiformes & & & Nematistius pectoralis Gill 1862 & $<0.1$ & $(\mathrm{tr} / \mathrm{st})-(\mathrm{cp})$ \\
\hline F. Fistulariidae & & & F. Coryphaenidae & & \\
\hline Fistularia commersonii Rüppell 1838 & $<0.1$ & (tr)-(sd) & Coryphaena hippurus Linneaus 1758 & $<0.1$ & (tr)-(op) \\
\hline Fistularia corneta Gilbert \& Starrks 1904 & $<0.1$ & (tr)-(sd) & F. Bramidae & & \\
\hline F. Syngnathidae & & & Bramidae type 1 & $<0.1$ & $-(\mathrm{mp})$ \\
\hline Syngnathus type 1 & $<0.1$ & $-(\mathrm{sd})$ & F. Lutjanidae & & \\
\hline O. Scorpaeniformes & & & Lutjanus argentiventris (Peters 1869) & $<0.1$ & (tr)-(sd) \\
\hline S.O. Scorpaenoidei & & & Lutjanus guttatus (Steindachner 1869) & $<0.1$ & (tr)-(sd) \\
\hline F. Scorpaenidae & & & Lutjanus novemfasciatus Gill 1862 & $<0.1$ & $(\operatorname{tr})-(\mathrm{sd})$ \\
\hline Sebastes macdonaldi & 0.2 & $(\mathrm{tm})-(\mathrm{dd})$ & Lutjanus peru (Nichols \& Murphy 1922) & $<0.1$ & (tr)-(sd) \\
\hline (Eigenmann \& Beeson 1893) & & & Lutjanus spp. & $<0.1$ & $(\mathrm{tr})-(\mathrm{sd})$ \\
\hline Sebastes spp. & $<0.1$ & $(\mathrm{tm} / \mathrm{sa})-$ & Lutjanidae type 1 & $<0.1$ & (tr)-(sd) \\
\hline Pontinus sierra (Gilbert 1890) & $<0.1$ & $(\mathrm{tr} / \mathrm{st})-(\mathrm{dd})$ & F. Malacanthidae & & \\
\hline Pontinus spp. & $<0.1$ & $(\operatorname{tr})-(\mathrm{sd})$ & Caulolatilus princeps (Jenyns 1842) & $<0.1$ & (wd)-(sd) \\
\hline Scorpaena guttata Girard 1854 & $<0.1$ & $(\mathrm{tm})-(\mathrm{sd})$ & F. Gerreidae & & \\
\hline Scorpaena spp. & $<0.1$ & $(\operatorname{tr} / \mathrm{st})-(\mathrm{dd})$ & Diapterus peruvianus (Sauvage 1879) & 0.1 & (tr)-(sd) \\
\hline Scorpaenodes xyris(Jordan \& Gilbert 1882) & $<0.1$ & (wd)-(sd) & Eucinostomus currani & $<0.1$ & $(\mathrm{tr} / \mathrm{st})-(\mathrm{sd})$ \\
\hline Sebastolobus altivelis Gilbert 1896 & $<0.1$ & $(\mathrm{st} / \mathrm{tm})-(\mathrm{dd})$ & Yáñez-Arancibia 1978 & & \\
\hline
\end{tabular}


TABLE 1 (Cont.). - Systematic list of the fish larvae collected in the Gulf of California during 1984-1988 with the percent abundance (\%). Faunistic affinity (FA): tropical (tr); subtropical (st); temperate (tm); subarctic (sa); wide distribution in the eastern Pacific (wd). Habitat (Hab): shallow demersal (sd); deep demersal (dd); coastal pelagic (cp); ocean epipelagic (op); mesopelagic (mp); and bathypelagic (bp).

\begin{tabular}{|c|c|c|c|c|c|}
\hline Taxon & $\%$ & $(\mathrm{FA})-(\mathrm{Hab})$ & Taxon & $\%$ & $(\mathrm{FA})-(\mathrm{Hab})$ \\
\hline Eucinostomus dowii (Gill 1863) & 0.1 & $(\mathrm{tr} / \mathrm{st})-(\mathrm{sd})$ & Hypsoblennius jenkinsi & $<0.1$ & $(\mathrm{tm})-(\mathrm{sd})$ \\
\hline Eucinostomus gracilis (Gill 1862) & 0.6 & $(\mathrm{tr})-(\mathrm{sd})$ & (Jordan \& Evermann 1896) & & \\
\hline Gerreidae type 1 & $<0.1$ & & Hypsoblennius spp. & $<0.1$ & $(\mathrm{tm})-(\mathrm{sd})$ \\
\hline F. Haemulidae & & & Ophioblennius steindachneri & $<0.1$ & (tr)-(sd) \\
\hline Anisotremus davidsoni (Steindachner 1875) & 0.2 & $(\mathrm{tm})-(\mathrm{sd})$ & Jordan \& Evermann 1898 & & \\
\hline Anisotremus spp. & $<0.1$ & (tr)-(sd) & Blenniidae type 1 & $<0.1$ & $-(\mathrm{sd})$ \\
\hline Orthopristis spp. & $<0.1$ & (tr)-(sd) & S.O. Gobioidei & & \\
\hline Xenistius californiensis (Steindachner 1875) & 0.2 & $(\mathrm{tr} / \mathrm{st})-(\mathrm{sd})$ & F. Eleotridae & & \\
\hline Haemulidae type 1 & $<0.1$ & & Eleotridae type 1 (Erotelis armiger?) & $<0.1$ & \\
\hline F. Sparidae & & & Eleotridae & 0.6 & \\
\hline Calamus brachysomus (Lockington 1880) & $<0.1$ & $(\mathrm{tr} / \mathrm{st})-(\mathrm{sd})$ & F. Gobiidae & & \\
\hline F. Sciaenidae & & & Coryphopterus type 1 & $<0.1$ & $-(\mathrm{sd})$ \\
\hline Menticirrhus undulatus (Girard 1858) & $<0.1$ & $(\mathrm{tr})-(\mathrm{sd})$ & Gillichthys mirabilis Cooper 1864 & $<0.1$ & $(\mathrm{tm})-(\mathrm{sd})$ \\
\hline Roncador stearnsii (Steindachner 1875) & $<0.1$ & $(\mathrm{tm} / \mathrm{st})-(\mathrm{sd})$ & Gobulus crescentalis (Gilbert 1892) & $<0.1$ & $(\mathrm{st})-(\mathrm{sd})$ \\
\hline Seriphus politus Ayres 1860 & $<0.1$ & $(\mathrm{tm})-(\mathrm{sd})$ & Ilypnus gilberti & $<0.1$ & $(\mathrm{tm})-(\mathrm{sd})$ \\
\hline Umbrina roncador Jordan \& Gilbert 1882 & $<0.1$ & (st)-(sd) & (Eigenmann \& Eigenmann 1889) & & \\
\hline Sciaenidae & $<0.1$ & & Lythrypnus dalli (Gilbert 1890) & 0.6 & $(\mathrm{tm})-(\mathrm{sd})$ \\
\hline F. Mullidae & & & Lythrypnus spp. & $<0.1$ & $-(\mathrm{sd})$ \\
\hline Mullidae type 1 & $<0.1$ & $-(\mathrm{sd})$ & Quietula y-cauda & $<0.1$ & $(\mathrm{tm})-(\mathrm{sd})$ \\
\hline F. Kyphosidae & & & (Jenkins \& Evermann 1889) & & \\
\hline Kyphosidae type 1 & $<0.1$ & $-(\mathrm{sd})$ & Gobiidae type 1 & $<0.1$ & $-(\mathrm{sd})$ \\
\hline F. Ephippidae & & & Gobiidae & 0.6 & $-(\mathrm{sd})$ \\
\hline Chaetodipterus zonatus (Girard 1858) & $<0.1$ & $(\mathrm{tr} / \mathrm{st})-(\mathrm{sd})$ & F. Microdesmidae & & \\
\hline Parapsettus panamensisSteindachner 1875 & $<0.1$ & $(\mathrm{tr})-(\mathrm{sd})$ & Clarkichthys bilineatus (Clark 1936) & $<0.1$ & $-(\mathrm{sd})$ \\
\hline F. Chaetodontidae & & & Microdesmidae type 1 & $<0.1$ & $-(\mathrm{sd})$ \\
\hline Chaetodontidae type 1 & $<0.1$ & $-(\mathrm{sd})$ & S.O Sphyraenoidei & & \\
\hline F. Pomacentridae & & & F. Sphyraenidae & & \\
\hline Abudefduf troschelii (Gill 1862) & $<0.1$ & $(\operatorname{tr})-(\mathrm{sd})$ & Sphyraena argentea Girard 1854 & $<0.1$ & $(\mathrm{tm})-(\mathrm{cp})$ \\
\hline Chromis type 1 & $<0.1$ & $(\mathrm{tr})-(\mathrm{sd})$ & Sphyraena ensis Jordan \& Gilbert 1882 & $<0.1$ & $(\mathrm{tr})-(\mathrm{cp})$ \\
\hline Chromis type 2 & $<0.1$ & $(\mathrm{tr})-(\mathrm{sd})$ & Sphyraena lucasana Gill 1863 & $<0.1$ & $(\mathrm{st})-(\mathrm{sd})$ \\
\hline Hypsypops rubicundus (Girard 1854) & $<0.1$ & $\mathrm{tm}-(\mathrm{sd})$ & S.O Scombroidei & & \\
\hline Stegastes rectifraenum (Gill 1862) & 0.1 & $(\mathrm{st})-(\mathrm{sd})$ & F. Gempylidae & & \\
\hline Pomacentridae type 1 & $<0.1$ & $-(\mathrm{sd})$ & Gempylus serpens Cuvier 1829 & $<0.1$ & $(\mathrm{tr} / \mathrm{st})-(\mathrm{mp})$ \\
\hline F. Opistognathidae & & & Gempylidae type 1 & $<0.1$ & $-(\mathrm{mp})$ \\
\hline Opistognathus spp. & $<0.1$ & $-(s d)$ & F. Scombridae & & \\
\hline F. Howellidae & & & Auxis spp. & 0.2 & (tr/st)-(op) \\
\hline Howella spp. & $<0.1$ & $-(\mathrm{mp})$ & Euthynnus lineatus Kishinouye 1920 & 0.1 & (tr)-(op) \\
\hline S.O. Mugiloidei & & & Sarda chiliensis (Cuvier 1832) & $<0.1$ & $(\mathrm{tm} / \mathrm{sa})-(\mathrm{cp})$ \\
\hline F. Mugilidae & & & Sarda type 1 & $<0.1$ & $-(\mathrm{cp})$ \\
\hline Mugil cephalus Linnaeus 1758 & $<0.1$ & $(\mathrm{tr} / \mathrm{st})-(\mathrm{cp})$ & Scomber japonicus Houttuyn 1782 & 1.4 & $(\mathrm{tm} / \mathrm{st})-(\mathrm{cp})$ \\
\hline Mugilidae type 1 & $<0.1$ & $(\mathrm{tr} / \mathrm{st})-(\mathrm{cp})$ & Thunnus spp. & $<0.1$ & (tr/st)-(op) \\
\hline S.O. Polynemoidei & & & F. Trichiuridae & & \\
\hline F. Polynemidae & & & Lepidopus fitchi Rosenblatt \& Wilson 198 & $7<0.1$ & (st)-(mp) \\
\hline Polydactylus approximans & $<0.1$ & $(\mathrm{tr} / \mathrm{st})-(\mathrm{sd})$ & Trichiurus nitens Garman 1899 & $<0.1$ & (wd)-(mp) \\
\hline (Lay \& Bennett 1839) & & & Trichiuridae type 1 & $<0.1$ & $-(\mathrm{mp})$ \\
\hline Polydactylus opercularis (Gill 1863) & $<0.1$ & $(\mathrm{tr} / \mathrm{st})-(\mathrm{sd})$ & S.O Stromateoidei & & \\
\hline S.O. Labroidei & & & F. Nomeidae & & \\
\hline F. Labridae & & & Cubiceps pauciradiatus Günther 1872 & $<0.1$ & $(\mathrm{tr})-(\mathrm{op})$ \\
\hline Halichoeres dispilus (Günther 1864) & $<0.1$ & $(\operatorname{tr})-(\mathrm{sd})$ & Cubiceps spp. & $<0.1$ & -(op) \\
\hline Halichoeres semicinctus (Ayres 1859) & $<0.1$ & $(\mathrm{tm})-(\mathrm{sd})$ & Psenes sio & $<0.1$ & (tr)-(op) \\
\hline Halichoeres spp. & $<0.1$ & $-(\mathrm{sd})$ & Psenes type 1 & $<0.1$ & $-(\mathrm{op})$ \\
\hline Oxyjulis californica (Günther 1861) & $<0.1$ & (tm)-(sd) & Nomeidae type 1 & $<0.1$ & \\
\hline Semicossyphus pulcher (Ayres 1854) & $<0.1$ & $(\mathrm{tm})-(\mathrm{sd})$ & F. Stromateidae & & \\
\hline Thalassoma spp. & $<0.1$ & (tr)-(sd) & Peprilus type 1 & $<0.1$ & $(\mathrm{tr})-(\mathrm{sd})$ \\
\hline Xyrichthys mundiceps Gill 1962 & $<0.1$ & $(\mathrm{tr})-(\mathrm{sd})$ & Stromateidae & $<0.1$ & $-(\mathrm{sd})$ \\
\hline Xyrichthys pavo (Valenciennes 1840$)$ & $<0.1$ & $(\mathrm{tr})-(\mathrm{sd})$ & O Pleuronectiformes & & \\
\hline Labridae & $<0.1$ & $-(\mathrm{sd})$ & S.O Pleuronectoidei & & \\
\hline F. Scaridae & & & F. Paralichthyidae & & \\
\hline Scarus spp. & $<0.1$ & $(\mathrm{tr})-(\mathrm{sd})$ & Citharichthys fragilis Gilbert 1890 & 0.2 & $(\mathrm{tm} / \mathrm{st})-(\mathrm{sd})$ \\
\hline S.O. Trachinoidei & & & Citharichthys gordae & $<0.1$ & $(\mathrm{tr})-(\mathrm{sd})$ \\
\hline F. Chiasmodontidae & & & Beebe \& Tee-Van 1938 & & \\
\hline Chiasmodon niger Johnson 1864 & $<0.1$ & $(\mathrm{tr} / \mathrm{st})-(\mathrm{mp})$ & Citharichthys platophrys Gilbert 1891 & $<0.1$ & $(\mathrm{tr})-(\mathrm{sd})$ \\
\hline Chiasmodontidae type 1 & $<0.1$ & $(\mathrm{tr} / \mathrm{st})-(\mathrm{mp})$ & Citharichthys spp. & $<0.1$ & $-(\mathrm{sd})$ \\
\hline S.O. Blennioidei & & & Cyclopsetta panamensis & $<0.1$ & $(\mathrm{tr})-(\mathrm{sd})$ \\
\hline F. Tripteygiidae & & & (Steindachner 1876) & & \\
\hline Tripteygiidae & $<0.1$ & $(\mathrm{tr} / \mathrm{st})-(\mathrm{sd})$ & Etropus crossotus Jordan \& Gilbert 1882 & $<0.1$ & $(\operatorname{tr})-(\mathrm{sd})$ \\
\hline F. Labrisomidae & & & Syacium ovale (Günther 1864) & 1.2 & (tr)-(sd) \\
\hline Labrisomus xanti (Gill 1860) & $<0.1$ & $(\mathrm{tr})-(\mathrm{mp})$ & Hippoglossina stomata & $<0.1$ & $(\mathrm{tm})-(\mathrm{sd})$ \\
\hline F. Blenniidae & & & Eigenmann \& Eigenmann 1890 & & \\
\hline Hypsoblennius gentilis (Girard 1854) & $<0.1$ & $(\mathrm{tm})-(\mathrm{sd})$ & Paralichthys californicus (Ayres 1859) & $<0.1$ & $(\mathrm{tm})-(\mathrm{sd})$ \\
\hline
\end{tabular}


TABLE 1 (Cont.). - Systematic list of the fish larvae collected in the Gulf of California during 1984-1988 with the percent abundance (\%). Faunistic affinity (FA): tropical (tr); subtropical (st); temperate (tm); subarctic (sa); wide distribution in the eastern Pacific (wd). Habitat (Hab): shallow demersal (sd); deep demersal (dd); coastal pelagic (cp); ocean epipelagic (op); mesopelagic (mp); and bathypelagic (bp).

\begin{tabular}{|c|c|c|c|c|c|}
\hline Taxon & $\%$ & (FA)-(Hab) & Taxon & $\%$ & $(\mathrm{FA})-(\mathrm{Hab})$ \\
\hline $\begin{array}{l}\text { Paralichthys woolmani } \\
\text { Jordan \& Williams in Gilbert } 1897\end{array}$ & $<0.1$ & $(\mathrm{tr})-(\mathrm{sd})$ & $\begin{array}{l}\text { Achirus mazatlanus (Steindachner 1869) } \\
\text { F. Cynoglossidae }\end{array}$ & $<0.1$ & $(\mathrm{tr} / \mathrm{st})-(\mathrm{sd})$ \\
\hline Xistreurys liolepis Jordan \& Gilbert 1880 & $<0.1$ & $(\mathrm{tm})-(\mathrm{sd})$ & Symphurus atricaudus & $<0.1$ & $(\mathrm{tm})-(\mathrm{sd})$ \\
\hline Paralichthyidae type 1 & $<0.1$ & $-(\mathrm{sd})$ & (Jordan \& Gilbert 1880) & & \\
\hline F. Bothidae & & & Symphurus williamsi & 0.5 & $(\operatorname{tr})-(\mathrm{sd})$ \\
\hline Bothus leopardinus (Günther 1862) & $<0.1$ & $(\operatorname{tr})-(\mathrm{sd})$ & (Jordan \& Culver 1895) & & \\
\hline $\begin{array}{l}\text { Engyophrys sanctilaurentii } \\
\text { Jordan \& Bollman } 1890\end{array}$ & $<0.1$ & $(\mathrm{tr})-(\mathrm{sd})$ & $\begin{array}{c}\text { Symphurus spp. } \\
\text { O Tetraodontiformes }\end{array}$ & 0.2 & $(\mathrm{tr})-(\mathrm{sd})$ \\
\hline Monolene asaedai Clark 1936 & $<0.1$ & $(\operatorname{tr})-(\mathrm{sd})$ & F. Balistidae & & \\
\hline Perissias taeniopterus (Gilbert 1890) & $<0.1$ & (tr)-(sd) & Balistidae type 1 (Balistes polilepis?) & 0.5 & $(\mathrm{tr} / \mathrm{st})-(\mathrm{sd})$ \\
\hline F. Pleuronectidae & & & F. Tetraodontidae & & \\
\hline Hypsopsetta guttulata (Girard 1856) & $<0.1$ & $(\mathrm{tm})-(\mathrm{sd})$ & Sphoeroides annulatus (Jenyns 1842) & $<0.1$ & $(\mathrm{tr} / \mathrm{st})-(\mathrm{sd})$ \\
\hline Pleuronichthys verticalis & $<0.1$ & $(\mathrm{tm})-(\mathrm{sd})$ & Sphoeroides lobatus (Steindachner 1870) & $<0.1$ & $(\mathrm{tr} / \mathrm{st})-(\mathrm{sd})$ \\
\hline Jordan \& Gilbert 1880 & & & Sphoeroides type 1 & $<0.1$ & $(\operatorname{tr} / \mathrm{st})-(\mathrm{sd})$ \\
\hline $\begin{array}{l}\text { S.O Soleoidei } \\
\text { F. Achiridae }\end{array}$ & & & Tetraodontidae type 1 & $<0.1$ & $-(\mathrm{sd})$ \\
\hline
\end{tabular}

according to their specific pigmentation patterns and morphometric characteristics, though some larvae could not be identified to species or to type owing to a lack of information or distinguishing characters, which means that these taxa (genus or family) could include one or more species.

Among the 95 families identified only eight exceeded $1 \%$ of the total capture; together these eight represented $88.5 \%$ of the total abundance (Table 2). Twenty species, including mesopelagic, epipelagic and demersal species, represented $90 \%$ of the total abundance (Table 3). The principal characteristic of the larval fish community in the Gulf of California was the dominance of mesopelagic forms, primarily of the families Myctophidae, Phosichthyidae and Bathylagidae, which represent-

TABLE 2. - Taxonomic list of families of fish larvae in the Gulf of California during 1984-1987, ranked by their relative abundance (\%).

\begin{tabular}{|c|c|c|c|c|c|c|c|c|}
\hline Rank & Taxa & $\%$ & Ran & Taxa & $\%$ & Rank & Taxa & $\%$ \\
\hline 1 & Myctophidae & 45.3 & 33 & Aulopidae & $<0.1$ & 65 & Mirapinnidae & $<0.1$ \\
\hline 2 & Engraulidae & 20.1 & 34 & Blenniidae & $<0.1$ & 66 & Moridae & $<0.1$ \\
\hline 3 & Clupeidae & 10.2 & 35 & Bramidae & $<0.1$ & 67 & Mugilidae & $<0.1$ \\
\hline 4 & Phosichthyidae & 6.8 & 36 & Bythitidae & $<0.1$ & 68 & Mullidae & $<0.1$ \\
\hline 5 & Bathylagidae & 1.7 & 37 & Carapidae & $<0.1$ & 69 & Muraenidae & $<0.1$ \\
\hline 6 & Scombridae & 1.7 & 38 & Chaetodontidae & $<0.1$ & 70 & Nematistiidae & $<0.1$ \\
\hline 7 & Paralichthyidae & 1.5 & 39 & Chiasmodontidae & $<0.1$ & 71 & Nemichthyidae & $<0.1$ \\
\hline 8 & Gobiidae & 1.2 & 40 & Coryphaenidae & $<0.1$ & 72 & Neoscopelidae & $<0.1$ \\
\hline 9 & Albulidae & 0.7 & 41 & Cyematidae & $<0.1$ & $7 \overline{3}$ & Nettastomatidae & $<0.1$ \\
\hline 10 & Carangidae & 0.7 & 42 & Derichthyidae & $<0.1$ & 74 & Nomeidae & $<0.1$ \\
\hline 11 & Cynoglossidae & 0.7 & 43 & Ephippidae & $<0.1$ & 75 & Notacanthidae & $<0.1$ \\
\hline 12 & Gerreidae & 0.7 & 44 & Exocoetidae & $<0.1$ & 76 & Ogcocephalidae & $<0.1$ \\
\hline 13 & Merlucciidae & 0.7 & 45 & Fistulariidae & $<0.1$ & 77 & Oneirodidae & $<0.1$ \\
\hline 14 & Eleotridae & 0.6 & 46 & Gempylidae & $<0.1$ & 78 & Opistognathidae & $<0.1$ \\
\hline 15 & Balistidae & 0.5 & 47 & Gigantactinidae & $<0.1$ & 79 & Paralepididae & $<0.1$ \\
\hline 16 & Haemulidae & 0.4 & 48 & Gonostomatidae & $<0.1$ & 80 & Pleuronectidae & $<0.1$ \\
\hline 17 & Serranidae & 0.4 & 49 & Hemiramphidae & $<0.1$ & 81 & Polynemidae & $<0.1$ \\
\hline 18 & Bregmacerotidae & 0.3 & 50 & Holocentridae & $<0.1$ & 82 & Priacanthidae & $<0.1$ \\
\hline 19 & Ophichthidae & 0.3 & 51 & Howellidae & $<0.1$ & 83 & Scaridae & $<0.1$ \\
\hline 20 & Scorpaenidae & 0.3 & 52 & Kyphosidae & $<0.1$ & 84 & Sciaenidae & $<0.1$ \\
\hline 21 & Argentinidae & 0.1 & 53 & Labridae & $<0.1$ & 85 & Scopelarchidae & $<0.1$ \\
\hline 22 & Bothidae & 0.1 & 54 & Labrisomidae & $<0.1$ & 86 & Serrivomeridae & $<0.1$ \\
\hline 23 & Congridae & 0.1 & 55 & Linophrynidae & $<0.1$ & 87 & Sparidae & $<0.1$ \\
\hline 24 & Elopidae & 0.1 & 56 & Lophiidae & $<0.1$ & 88 & Sphyraenidae & $<0.1$ \\
\hline 25 & Ophidiidae & 0.1 & 57 & Lutjanidae & $<0.1$ & 89 & Stomiidae & $<0.1$ \\
\hline 26 & Pomacentridae & 0.1 & 58 & Macrouridae & $<0.1$ & 90 & Stromateidae & $<0.1$ \\
\hline 27 & Synodontidae & 0.1 & 59 & Malacanthidae & $<0.1$ & 91 & Syngnathidae & $<0.1$ \\
\hline 28 & Triglidae & 0.1 & 60 & Melamphaidae & $<0.1$ & 92 & Tetraodontidae & $<0.1$ \\
\hline 29 & Achiridae & $<0.1$ & 61 & Melanocetidae & $<0.1$ & 93 & Trachipteridae & $<0.1$ \\
\hline 30 & Antennariidae & $<0.1$ & 62 & Melanostomiidae & $<0.1$ & 94 & Trichiuridae & $<0.1$ \\
\hline 31 & Apogonidae & $<0.1$ & 63 & Microdesmidae & $<0.1$ & 95 & Tripterygiidae & $<0.1$ \\
\hline 32 & Atherinidae & $<0.1$ & 64 & Microstomatidae & $<0.1$ & & & \\
\hline
\end{tabular}




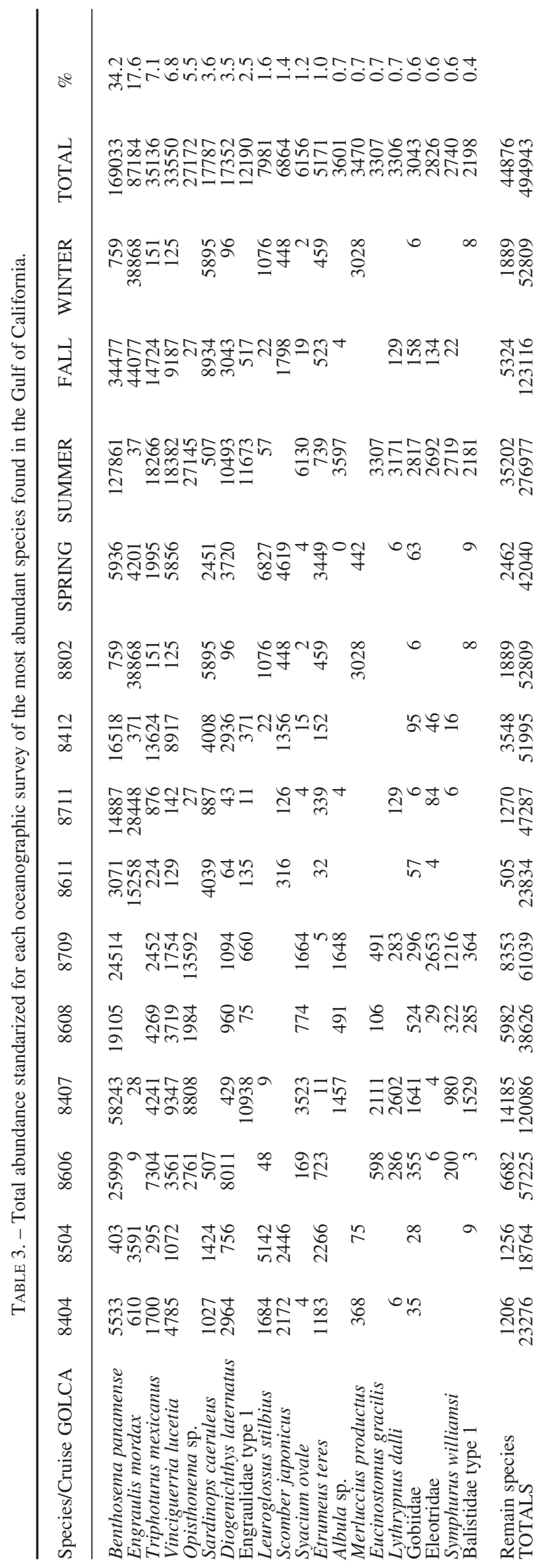

ed almost $54 \%$ of the abundance, but the coastal pelagic families Clupeidae, Engraulidae and Scombridae contributed $32 \%$ to the total catch (Table 2).

Winter had the fewest species (33) (Table 4), with a high abundance of Engraulis mordax and Sardinops caeruleus (Table 3). Accordingly, coastal pelagic species were the most abundant component of the ichthyoplankton (63\%) (Table 4), as were species with temperate affinity (96\%) (Table 5). In contrast, summer had the highest number of species (271), mostly with tropical-subtropical affinity (73\%). The number of shallow demersal species was highest during the summer (130), but the most abundant taxa were mesopelagic (mainly Benthosema panamense), representing the $65 \%$ of the total catch (Table 4). Fall and spring had more tropical/subtropical species (58 and 59\%) than temperate species (27 and 28\%), however, the abundance of tropical/subtropical species and that of temperate species was nearly similar $(\approx 40 \%)$. Mesopelagic taxa again were the most abundant group during fall and spring, but their dominance over the next most abundant group (coastal pelagics) was not as pronounced as during winter and summer (Table 4). For example, the mesopelagic Leuroglossus stilbius was the most abundant species during spring with $16 \%$ of the total catch, and the coastal pelagic E. mordax was first during fall with $11 \%$. In contrast, E. mordax was the most abundant species during winter with $74 \%$ of the total (Table 3).

\section{DISCUSSION}

The taxonomic list of fish larvae in the Gulf of California given here is the most extensive yet presented. The 283 taxa identified represent approximately $38 \%$ of the 753 species recorded as adults in the Gulf (Castro-Aguirre et al., 1995); however, the list is undoubtedly conservative because at least 32 of the taxa could include more than one species, and the selectivity of the sampling technique did not allow catches of some species from shallow waters (e.g. Haemulidae and Achiridae).

The Gulf of California represents one of the most diverse ecosystems in the Eastern Pacific (CastroAguirre et al., 1995) and this diversity is reflected in the collections of fish larvae obtained in this work. In contrast with the 283 taxa identified from the Gulf of California, much more extensive samplings made in the California Current contained only 249 larval fish taxa in 31,214 samples (Moser and Smith, 
TABLE 4. - Seasonal larval abundance and number of species by habitat: (cp) coastal pelagic; (op) ocean epipelagic; (mp) mesopelagic; (bp) bathypelagic; (sd) shallow demersal and (dd) deep demersal; (nd) not determined. Numbers in parenthesis are the percentage by season.

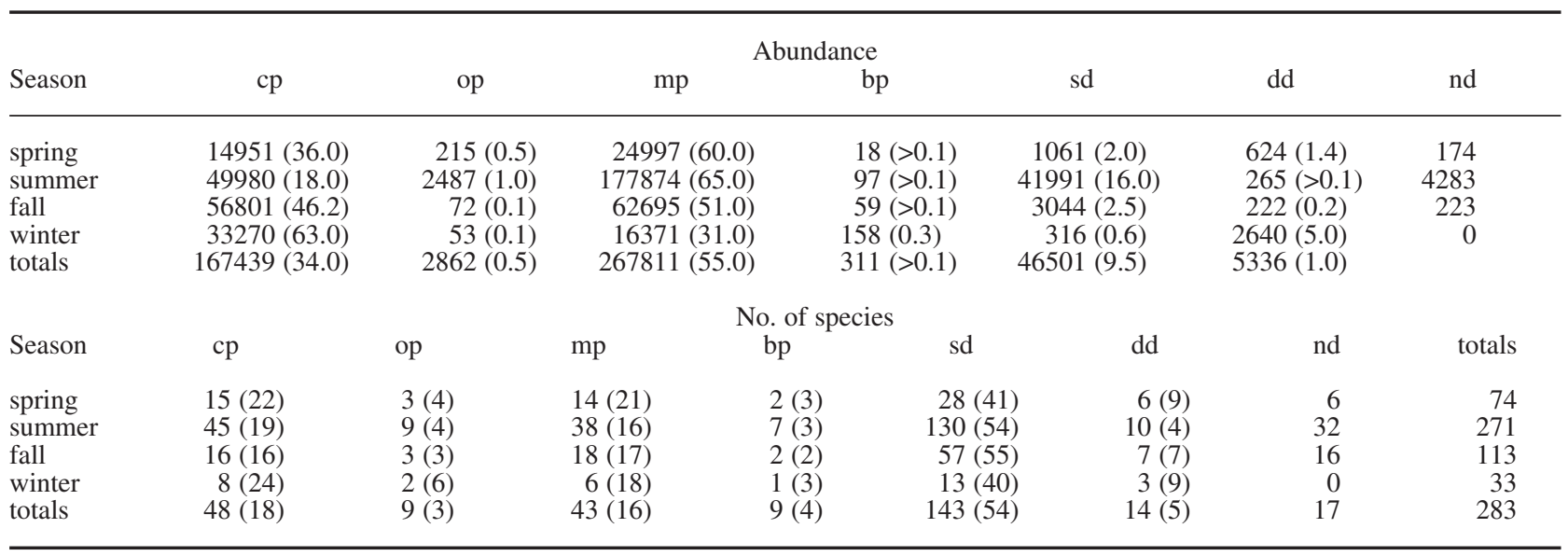

TABLE 5. - Seasonal larval abundance and number of species by faunal affinity: (Tr-SbTr) tropical/subtropical; (Trans) transitional; (Tm) temperate; (Wd) wide distribution and (nd) not determined. Numbers in parenthesis are the percentage by season.

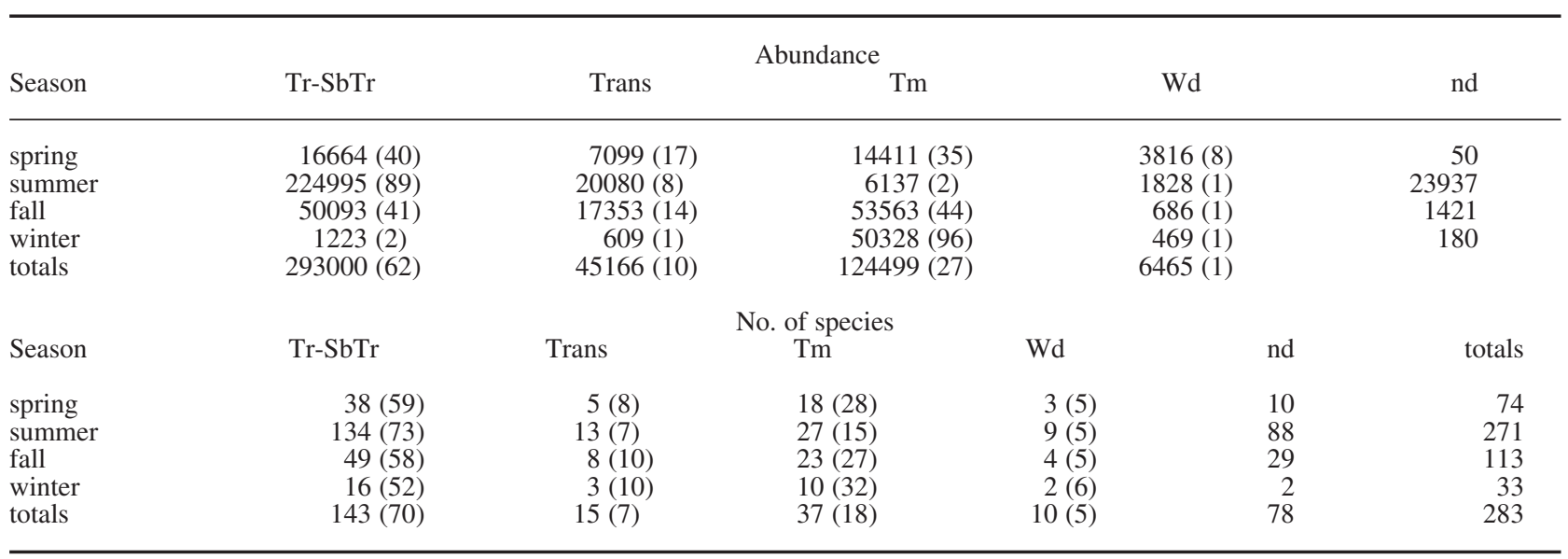

1993). Less exhaustive studies showed the presence of 214 taxa in the northern region of the west coast of Baja California (Jiménez-Rosenberg et al., 2000), and 102 taxa from 132 samples south of the Gulf, in the Jalisco and Colima area (Franco-Gordo et al., 1999). At a higher taxonomic level, Aguilar-Ibarra and Vicencio-Aguilar (1994) report 37 families in the vicinity of the Costa Rica Dome, Ahlstrom (1972) reported 56 families in a larger survey of the Eastern Tropical Pacific, and Moser et al. (1974) reported 50 families during 1956-1957 in the Gulf of California compared with 95 found in this work.

Diversity differences at the family level between this study and Moser's could reflect a lack of sampling during summer because we found a large increase in the number of species during this season, but differences in the species composition and abundance were also evident, even within seasons. The most abundant species during 1956-1957 in the Gulf of California were Vinciguerria lucetia and Bregmaceros bathymaster (Table 6; Moser et al., 1974), and B. panamense, L. stilbius and Engraulidae were found with the lowest abundances. In contrast, during 1984-1987, B. panamense and E. mordax were the most abundant species and $B$. bathymaster was ranked $27^{\text {th }}$, with less than $0.3 \%$ of the total catch. Mesopelagic taxa contributed $39 \%$ of the total catch in 1956-57 compared with $55 \%$ in $1984-87$, while for coastal pelagic taxa the values were 22 and $34 \%$ respectively.

Engraulis mordax larvae were absent in the Gulf of California during at least 1972 (Moser et al., 1974), but in 1984, two years before the unexpected high commercial fishery catch of northern anchovy in this region, this species was one of the most abundant. The high catch of this species in 1986, accompanied by a dramatic decline in the sardine catch and the analyses of scales in laminar sediments of the 
TABLE 6. - Rank and percetage contribution for the 25 most abundant taxa in the Gulf of California during 1956-1957 (Moser et al., 1974).

\begin{tabular}{|c|c|c|c|c|c|}
\hline Rank & Species & $\%$ & Rank & Species & $\%$ \\
\hline 1 & Vinciguerria lucetia & 21.9 & 14 & Serranidae & 1.5 \\
\hline 2 & Bregmaceros bathymaster & 19.0 & 15 & Disintegrated & 1.5 \\
\hline 3 & Opisthonema spp. & 9.2 & 16 & Engraulidae & 1.4 \\
\hline 4 & Triphoturus mexicanus & 7.5 & 17 & Etrumeus teres & 1.1 \\
\hline 5 & Scomber japonicus & 6.5 & 18 & Scorpaenidae & 1.0 \\
\hline 6 & Diogenichthys laternatus & 5.1 & 19 & Gerridae & 0.8 \\
\hline 7 & Sardinops sagax caeruleus & 3.2 & 20 & Auxis rochei & 0.7 \\
\hline 8 & Benthosema panamense & 2.9 & 21 & Carangidae & 0.7 \\
\hline 9 & Unidentified & 2.7 & 22 & Gobiidae & 0.7 \\
\hline 10 & Leuroglossus stilbius & 2.0 & 23 & Pomadasyidae & 0.5 \\
\hline 11 & Trichiuridae & 2.0 & 24 & Synodus spp. & 0.5 \\
\hline 12 & Bothidae & 1.7 & 25 & Symphurus spp. & 0.4 \\
\hline 13 & Sciaenidae & 1.5 & & All others & 4 \\
\hline
\end{tabular}

Gulf, proved that contrary to the general agreement that the northern anchovy did not occur in the Gulf of California (Ahlstrom, 1967), E. mordax as well as mackerel, myctophids and sardine have cycles of several decades of high and low abundance (Holmgren-Urba and Baumgartner, 1993) that are associated with climate change and have strong effects on the ecosystem structure. Similar changes were observed in the abundance of fish larvae in the California Current region during a cool regime from 1950-1975 and a warm regime from 1975-2000 (Moser et al., 2001). In the Gulf of California the warm regime apparently began in 1982-1983, as recorded in several physical variables of the sea and the atmosphere (Bernal et al., 2001), but effects on the ichthyoplankton community have not been reported. The presence of E. mordax as one of the most abundant fish larvae during spring 1984 and 1985 may reflect this regime change, and the same may be true for the changes in abundance of $B$. panamense, B. bathymaster and L. stilbius.

Engraulis mordax and B. panamense, as well as the increase in the relative proportions of mesopelagic and epipelagic taxa, seem to characterise the warm regime in the Gulf of California. Unfortunately, because most taxa were not identified to species during the cool regime, we cannot address possible changes in faunal affinities except to note that on the basis of the most abundant species, both the cool and warm regime have a strong tropical/subtropical faunal component with seasonal changes in abundance and species composition.

In general, during 1984-1987 the larval fish community of the Gulf of California had a strong tropical component in both species composition and abundance (70 species and $62 \%$ of the total larvae), mirroring the faunal affinities of the adults inhabit- ing the area (Walker, 1960; Thompson et al., 1979; Briggs, 1974; Castro-Aguirre et al., 1995). Seasonal variability, however, shows contrasting changes between a well-developed tropical community during summer, clearly dominated by mesopelagic taxa, and a more temperate community during winter, dominated by coastal pelagic forms, but with lower species richness with transitional periods in spring and fall that nearly have a co-dominance between tropical-subtropical and temperate taxa (but with the mesopelagic as the more abundant group during spring). Another general characteristic of the larval fish community is the species richness of the shallow demersal taxa that represent almost $50 \%$ of the total species all year round, which is especially high during summer.

Seasonal changes in species composition and abundance in both faunal and habitat affinities reflect the sharp seasonal climate changes that exist between winter and summer in the Gulf of California. Sea surface temperatures reported between 1983-1996 in the northern Gulf range from 9 to $38^{\circ} \mathrm{C}$ between winter and summer, while in the south seasonal variation is less dramatic, ranging from 22 to $31^{\circ} \mathrm{C}$ (Soto-Mardones et al., 1999). Seasonal changes in the hydrography of the Gulf provide suitable environments for fishes that allow them to share the Gulf as a reproduction area. Tropical Pacific water, a warm water mass of low salinity $(34.65 £ \%$ o $\geq 34.85$ ), reaches the southern limit of the Gulf during winter, but during spring it flows north along the east coast (Alvarez-Borrego, 1983), so that by midsummer the whole area has tropical conditions (Rosas-Cota, 1977). This allows the reproduction of tropical-subtropical shallow demersal species, and promotes the dominance of mesopelagic species with epipelagic larval stages from the eastern tropical Pacific that were observed in our summer sam- 
ples. During autumn, tropical Pacific water starts to recede southward, decreasing the sea surface temperature, and the relict temperate/subarctic fauna starts its reproductive period so that in winter the larvae of temperate species become dominant. Coastal upwelling enhances the nutrient concentration and permits increased primary productivity of the area, which in turn supports the increased number of coastal pelagic species that was observed.

General intra- and inter-annual changes observed in the ichthyoplankton species composition since the first studies in the Gulf of California reflect a close relationship with the environmental changes that occurred. The appearance of larvae of species like E. mordax, before adults were recorded in the Gulf of California, as well as the changes in abundance of several species, show that ichthyoplankton studies are useful to make predictions about changes in the fisheries of this semi-enclosed ecosystem and that the analysis of the entire community can give us more information about the changes in the ecosystem. How these changes occur in space and time in the larval fish community is a second task of our future work.

\section{ACKNOWLEDGEMENTS}

We express our sincere thanks to the Centro Interdisciplinario de Ciencias Marinas of the Instituto Politécnico Nacional (CICIMAR-IPN) and the Coordinación General de Postgrado e Investigación (CGPI) for the financial support through the projects DEPI86804, DEPI868043 and DEPI903388. The first author wishes to thank the Consejo Nacional de Ciencia y Tecnología (CONACyT), Programa Institucional para la formación de Investigadores (PIFIIPN) and Teléfonos de México (Telmex) for the grant during his PhD stay at CICIMAR-IPN. S.P.A. Jiménez-Rosenberg, A. Hinojosa-Medina and R. Funes-Rodriguez wish to thank the Comisión de Operación y Fomento de Actividades Académicas (COFAA-IPN).

\section{REFERENCES}

Aguilar-Ibarra, A. and M.E. Vicencio-Aguilar. - 1994. Lista sistemática de las larvas y juveniles de peces en la región del Domo de Costa Rica. Rev. Biol. Trop., 42(3): 747-750.

Ahlstrom, E.H. - 1967. Co-occurrence of sardine and anchovy larvae in the California Current region, off California and Baja California. CalCOFI Rep., 11: 117-135.
Ahlstrom, E.H. - 1972. Kinds and abundance of fish larvae in the eastern tropical Pacific on the second multivessel EASTROPAC survey, and observations on the annual cycle of larval abundance. Fish. Bull., 70(4): 1153-1242.

Alvarez-Borrego, S. - 1983. Gulf of California. In: B.H. Ketchum (ed.), Estuaries and enclosed seas. pp. 427-449. Elsevier Scientific Publishing Co., Netherlands

Bernal, G., P. Ripa and J.C. Herguera. - 2001. Variabilidad oceanográfica y climática en el bajo Golfo de California: Influencias del trópico y del Pacífico norte. Cienc. Mar., 27(4): 595-617.

Briggs, J.C. - 1974. Marine zoogeography. McGraw Hill Book Co., EUA.

Castro-Aguirre, J.L., E.F. Balart and J. Arvizu-Martínez. - 1995. Contribución al conocimiento del origen y distribución de la ictiofauna del Golfo de California, México. Hidrobiol., 5(1-2): 57-78.

Ekman, S. - 1953. Zoogeography of the sea. Sidwick and Jackson Ltd., London.

Eschmeyer, W.M., H. Hamman and K.P. Smith. - 1983. Pacific coast fishes. Houghton Mifflin Co., New York.

Franco-Gordo, C., R. Flores-Vargas, C. Navarro-Rodríguez, R. Funes-Rodríguez and R. Saldierna-Martínez. - 1999. Ictioplancton de las costas de Jalisco y Colima, México (Diciembre de 1995 a Diciembre de 1996). Cienc. Mar., 25(1): 107-118.

Green-Ruiz, Y. and A. Hinojosa-Corona. - 1997. Study of the spawning area of the northern anchovy in the Gulf of California from 1990 to 1994 , using satellite images of the sea surface temperatures. J. Plankton Res., 19(8): 957-968.

Holmgren-Urba, D. and T.R. Baumgartner. - 1993. A 250 year story of pelagic fish abundances from the anaerobic sediments of the central Gulf of California. CalCOFI Rep., 34: 60-68

Jiménez-Rosenberg, S.P.A., G. Aceves-Medina, R. Avendaño-Ibarra, A. Hinojosa-Medina, S. Camarillo-Coop, R.J. SaldiernaMartínez, R. Funes-Rodríguez, M.E. Hernández-Rivas and T.R. Baumgartner-McBride. - 2000. Ictioplancton de la región sureña de la Corriente de California durante el fenómeno de El Niño septiembre 1997-octubre 1998. Comunicaciones Académicas. Serie Ecológica. CICESE, México.

Kendall, A.W., Jr. and A.C. Matarese. - 1994. Status of early life history descriptions of marine teleosts. Fish. Bull., 92: 725-736.

Loeb, V.J., P.E. Smith and H.G. Moser. - 1983. Ichthyoplankton and zooplankton abundance patterns in the California current area, 1975. CalCOFI Rep., 24: 109-164.

Moser, H.G. - 1996. The early stages of fishes in the California Current region. CalCOFI ATLAS No. 33. Allen Press Inc. Lawrence, Kansas.

Moser, H.G. and P.E. Smith. - 1993. Larval fish assemblages in the California Current region and their horizontal and vertical distributions across a front. Bull. Mar. Sci., 53(2): 645-691.

Moser, H.G., E.H. Ahlstrom., D. Kramer and E.G. Stevens. - 1974. Distribution and abundance of fish eggs and larvae in the Gulf of California. CalCOFI Rep., 17: 122-128.

Moser, H.G., P.E. Smith and L.E. Eber. - 1987. Larval fish assemblages in the California Current region, 1954-1960, a period of dynamic environmental change. CalCOFI Rep., 28: 97-127.

Moser, H.G., R.L. Charter, P.E. Smith, D.A. Ambrose, W. Watson, S.R. Charter and E.M. Sandknop. - 2001. Distributional atlas of fish larvae and eggs in the southern California Bight region: 1951-1998. CalCOFI Atlas No. 34. 166 pp.

Richards, W.J. - 1985. Status of the identification of the early life stages of fishes. Bull. Mar. Sci., 37(2): 756-760.

Rosas-Cota, A. - 1977. Corrientes geostróficas en el Golfo de California en la superficie y a $200 \mathrm{~m}$, durante las estaciones de invierno y verano. CalCOFI Rep., 19: 89-106.

Smith, P.E. and S.L. Ricardson. - 1979. Técnicas modelo para prospecciones de huevos y larvas de peces pelágicos. FAO Doc. Tec. Pesca 175, 107 pp.

Soto-Mardones, L., S.G. Marinone and A. Parés-Sierra. - 1999. Variabilidad espacio temporal de la temperatura superficial del mar en el Golfo de California. Cienc. Mar., 25(1): 1-30.

Thomson, D.A., L.T. Findley and A.N. Kerstitch. - 1979. Reef fishes of the sea of Cortez. John Wiley and Sons, New York.

Walker, B.W. 1960. - The distribution and affinities of the marine fish fauna of the Gulf of California. Syst. Zool., 9(3): 123-133.

Scient. ed.: J.J. Govoni 